\title{
LIXIVIACIÓN Y SECADO DE HOJUELAS DE NIM (Azadirachta indica) PARA OBTENER UN INSECTICIDA BOTÁNICO
}

\section{LEACHING AND DRYING OF NEEN FLAKES (Azadirachta indica) TO OBTAIN A BOTANICAL INSECTICIDE}

\author{
Malin Nyberg ${ }^{1}$, Apolinar Picado ${ }^{2, *}$, Rafael Gamero², Joaquín Martínez ${ }^{1}$ \\ ${ }^{1}$ Department of Chemical Enginering and Technology, KTH Royal Institute of Technology \\ Teknikringen 42, SE-100 44 Stockholm, Sweden \\ ${ }^{2}$ Facultad de Ingeniería Química, Universidad Nacional de Ingeniería (UNI) \\ Av. Universitaria, Managua 11127, Nicaragua \\ E-Mail*: picado@kth.se
}

(recibido/received: 16-Enero-2015; aceptado/accepted: 20-Marzo-2015)

\begin{abstract}
RESUMEN
El objetivo de este trabajo es estudiar experimentalmente la viabilidad de mejorar el procesamiento de hojuelas de nim en columnas, mediante la realización de la lixiviación del insecticida sin la remoción intermedia del solvente no-polar; y mediante la simultánea realización de ambas etapas de lixiviación. El secado de los solventes remanentes en las hojuelas de nim también se estudió experimentalmente. En la parte de lixiviación, los parámetros estudiados fueron tiempo de lixiviación, grosor de hojuelas, relación de volumen de solventes y volumen total de solventes. Estos parámetros mostraron tener una gran influencia en el rendimiento de los compuestos insecticidas, pero la influencia de la relación de solventes fue la más importante. Se demostró que más metanol que hexano tuvo que ser empleado para obtener un mayor rendimiento de compuestos insecticidas. El rendimiento de aceite fue influenciado principalmente por el grosor de las hojuelas. En la parte de secado, la temperatura del flujo de aire entrante tiene influencia en el proceso. Conclusiones sobre el tiempo de secado o la velocidad de secado no son posibles de realizar a partir de los resultados de los experimentos. Las variaciones en el tiempo de secado son demasiadas grandes y la velocidad de secado parece ser independiente de las condiciones de la etapa de lixiviación anterior.
\end{abstract}

Palabras clave: Lixiviación; Secado; Hojuelas de Nim; Insecticida

\begin{abstract}
The aim of this work is to experimentally study the feasibility of improving the processing of neem flakes in columns by performing the leaching of insecticide without intermediate removal of the non-polar solvent, and by performing simultaneously both leaching steps. The drying of the solvents remaining in the neem flakes is also experimentally studied. In the leaching part, the parameters studied were leaching time, thickness of the flakes, volume ratio of solvents, and total volume of solvents. These parameters showed to have a great influence on the yield of insecticide components, but the influence of solvent ratio was the most important one. It was shown that more methanol than hexane had to be used to obtain a large yield of insecticide compounds. The yield of oil was mainly influenced of the flake thickness. In the drying part, the temperature of the incoming airflow has influence on the process. Conclusions about the drying time or the drying rate are not possible to make from the results of the experiments. The variations in drying time are too large and the drying rate seems to be independent of the conditions of the previous leaching step.
\end{abstract}

Keywords: Leaching; Drying; Neem flakes; Insecticide 


\section{INTRODUCCIÓN}

En Nicaragua, la agricultura es el principal rubro que contribuye al producto interno bruto (PIB). A fin de reducir pérdidas en la producción, nuestros agricultores han utilizados insecticidas sintéticos por varios años. Esto a provocado efectos negativos tanto en la salud humana como en el medio ambiente, ya que debido a la estabilidad de estos compuestos se han acumulados en el suelo, plantas, animales, fuentes de aguas frescas y en el cuerpo humano. Además, los insecticidas tradicionalmente usados afectan indiscriminadamente a insectos nocivos como a organismos favorables. Otro problema es que muchos insectos han ganado resistencia en contra de estas sustancias durante los años (Espinosa, 2000).

Por las razones anteriores, es deseable encontrar un insecticida natural biodegradable que pueda ser producido localmente con un mínimo efecto sobre el medio ambiente. Un producto que ha cobrado popularidad es el insecticida obtenido a partir del árbol de nim (Azadirachta indica A. Juss), nativo de la India. Este árbol es conocido por sus propiedades medicinales en humanos (Biswas et al., 2002) y por su uso en control de plagas en cultivos agrícolas (Koul et al., 1990).

En los últimos años, el interés en el árbol de nim ha sido enfocado en sus propiedades repelentes contra insectos. La semilla de nim contiene aceite y una mezcla de diferentes sustancias con propiedades insecticidas. La sustancia más estudiada y la cual se cree que tiene el mayor efecto inhibidor en el crecimiento de insectos es la azadiractina A (Angulo-Escalante et al., 2004), de allí el principal interés de utilizar sus subproductos en la formulación de insecticidas biodegradables. La extracción del insecticida a partir de las semillas de nim se realiza en dos etapas de lixiviación. La primera, el aceite es lixiviado con un solvente no-polar, e.g., hexano; y luego el insecticida es lixiviado con un solvente polar, e.g., etanol. Entre las etapas de lixiviación y después de la recuperación del insecticida, los solventes deben ser removidos de los sólidos agotados. Este proceso puede ser por lote o semi-continuo.

En el proceso por lote se utilizan semillas de nim molidas, las cuales son lixiviadas en tanques agitados. Se usan semillas molidas para obtener una superficie interfacial máxima entre los sólidos y los solventes, además de una trayectoria de difusión corta para el soluto ya que estos factores mejoran la separación. En el proceso semi-continuo se utilizan hojuelas de nim en vez de semillas molidas. En este caso, las etapas de lixiviación se pueden realizar en columnas. Al no tener partes móviles, las columnas son más simples y fáciles de sellar a fin de evitar fuga de solvente como en los tanques agitados. Una ventaja adicional es que debido a la favorable fluidodinámica en el lecho de hojuelas, el aire es capaz de fluir a través del material y realizar en el mismo equipo la remoción los solventes después de las etapas de lixiviación.

Diversos estudios se han realizados con hojuelas de nim. Martínez et al. (1998) estudiaron la cinética de disolución del aceite e insecticida en hojuelas de semilla de nim aisladas. Persson y Sjöberg (1998) estudiaron las características hidrodinámicas y los parámetros de transporte en lechos que consistían de hojuelas de semilla de nim. En este estudio, se reportaron que los grosores de $0.7,1.3$ y $2 \mathrm{~mm}$ son los más apropiados para los experimentos de lixiviación y secado. Martínez (2003) y Martínez-Pavón (2006) realizaron experimentos de lixiviación con flujo de solvente ascendente y descendente en columnas empacadas con hojuelas de nim a diferentes temperaturas y grosores de hojuelas.

El objetivo de este trabajo es estudiar experimentalmente la viabilidad de mejorar el procesamiento de las hojuelas de nim en columnas realizando la lixiviación del insecticida sin remoción intermedia del solvente no-polar y realizando simultáneamente ambas etapas de lixiviación. Para los experimentos se utilizarán como solventes el hexano y metanol. Estudiando como las diferentes variables afectan el rendimiento del aceite y de la mezcla insecticida y comparando el rendimiento con estudios previos será posible evaluar si este método es apropiado. El trabajo incluye además el estudio experimental del secado en lecho fijo de los solventes remanentes en las hojuelas de nim. 


\section{MATERIALES Y MÉTODOS}

\section{Materiales}

Las semillas de nim utilizadas en los experimentos fueron obtenidas a través de la empresa COPYNIM, Managua, Nicaragua. Las semillas fueron descascarilladas y seleccionadas para luego ser convertidas en hojuelas utilizando un molino de rodos. Los grosores obtenidos para las hojuelas fueron de 0.7 y $2 \mathrm{~mm}$.

En los experimentos se utilizó hexano comercial para la extracción del aceite y metanol $(99.8 \%)$ para la extracción de la mezcla insecticida.

\section{Equipos}

\section{a) Lixiviación}

La operación de lixiviación se realizó en un soxhlet conectado a un condensador, a un balón de $500 \mathrm{~mL}$ y a un baño térmico que mantuvo la temperatura del agua de enfriamiento a $16{ }^{\circ} \mathrm{C}$. Además, un manto calefactor se utilizó para calentar los solventes (ver Fig. 1).

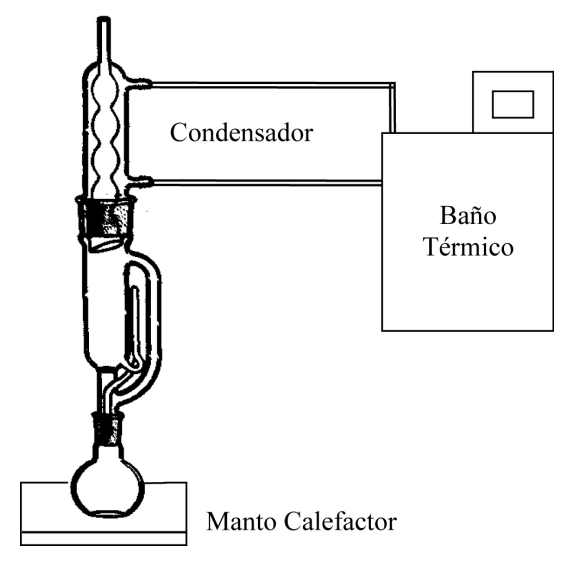

Fig. 1 Equipo experimental para la lixiviación.

b) Secado

Para realizar el secado de las hojuelas agotadas, estas se colocaron en una columna de PVC con un diámetro interno de $55 \mathrm{~mm}$ y una altura de $180 \mathrm{~mm}$. En el fondo de la columna (DC) se colocó una red para sostener el lecho de hojuelas. Aire con condiciones controladas fue introducido por el fondo de la columna. El aire fue tomado de la atmósfera por un ventilador (B) y se condujo a través de una columna de adsorción (AC) para reducirle la humedad y después se calentó pasándolo a través de una sección tubular con una resistencia eléctrica (ER). El flujo de aire caliente y seco, fluyó a través de la columna (DC) secando las hojuelas. El flujo de aire se reguló utilizando un inversor de frecuencia (FI) conectado a una fuente de poder (PS). Una muestra del flujo de salida fue, con intervalos de tiempo regular, tomada y enviada a un espectrofotómetro (SP) para analizar la composición del hexano y metanol durante todo el experimento de secado (ver Fig. 2).

La temperatura del aire fue controlada por un lazo de control automático (CT y TC) que regula la energía suministrada a la resistencia eléctrica (ER). Las temperaturas de entrada y salida en la columna fueron medidas utilizando termopares (IT y UT) conectados a una computadora (C). 


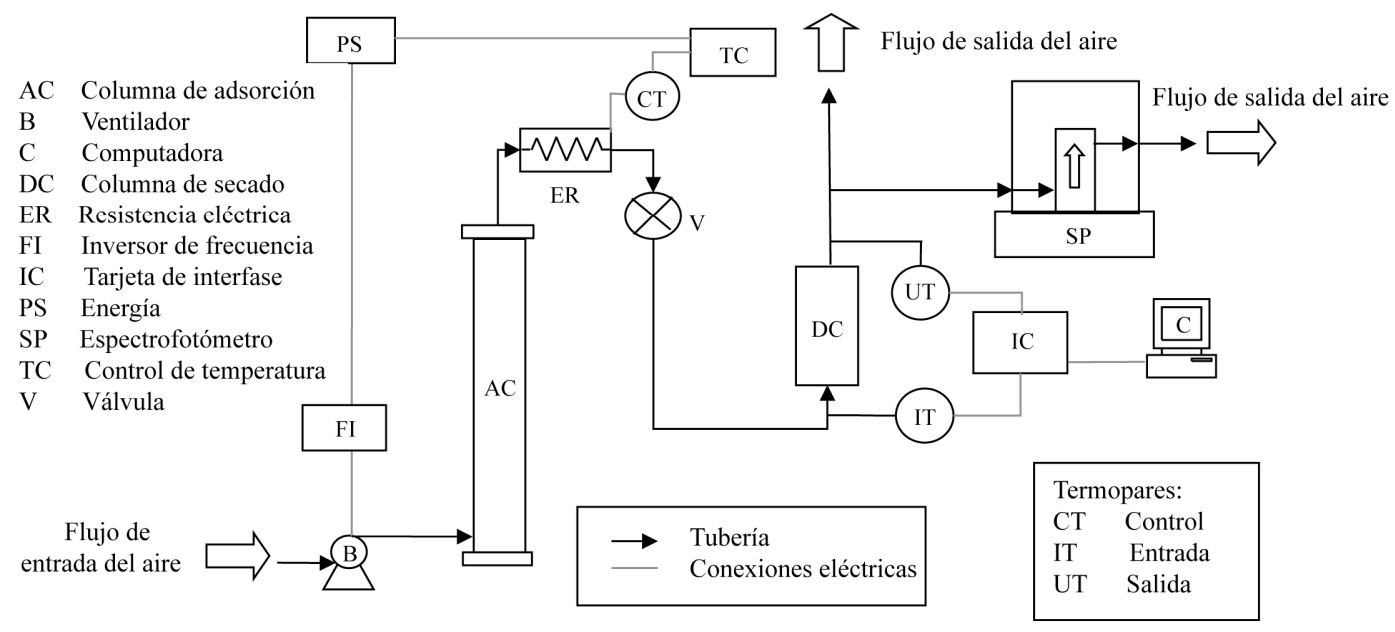

Fig. 2 Equipo experimental para el secado.

\section{Procedimientos}

En los experimentos, diversas variables fueron estudiadas a fin de evaluar sus efectos en los procesos de lixiviación y secado. Las variables estudiadas fueron: grosor de las hojuelas, relación de volumen de los solventes, volumen total de solventes, tiempo de lixiviación y temperatura de secado. En cada experimento se utilizaron $70 \mathrm{~g}$ de hojuelas de nim.

\section{a) Lixiviación}

La red que contenía a las hojuelas fue colocada en el soxhlet y los solventes en el balón. Los solventes, calentados por el manto calefactor, se evaporaron en dirección al condensador, donde son condensados. El fondo del soxhlet capturó a los solventes condensados hasta que el nivel del líquido en el soxhlet rebasó el nivel superior del tubo externo más pequeño. Entonces, los solventes cayeron nuevamente al balón por la fuerza capilar; esto continúo por 10, 20 o $30 \mathrm{~h}$. En la Tabla 1 se listan las condiciones utilizadas en la lixiviación.

Tabla 1 Resumen de las condiciones utilizadas en la lixiviación.

\begin{tabular}{cccc}
\hline $\begin{array}{c}\text { Tiempo } \\
\text { de } \\
\text { lixiviación } \\
(\mathrm{h})\end{array}$ & $\begin{array}{c}\text { Grosor de } \\
\text { la hojuela } \\
(\mathrm{mm})\end{array}$ & $\begin{array}{c}\text { Relación de } \\
\text { volumen de los } \\
\text { solventes } \\
\text { (hexano:metanol) }\end{array}$ & $\begin{array}{c}\text { Volumen } \\
\text { total de } \\
\text { solventes } \\
(\mathrm{mL})\end{array}$ \\
\hline 10 & 2.0 & $1: 1$ & 300 \\
10 & 2.0 & $1: 2$ & 300 \\
10 & 2.0 & $2: 1$ & 300 \\
20 & 2.0 & $1: 1$ & 300 \\
20 & 2.0 & $1: 2$ & 300 \\
20 & 2.0 & $1: 2$ & 450 \\
20 & 2.0 & $3: 4$ & 350 \\
20 & 2.0 & $1: 3$ & 400 \\
30 & 2.0 & $1: 2$ & 450 \\
30 & 2.0 & $1: 3$ & 400 \\
20 & 0.7 & $1: 2$ & 300 \\
20 & 0.7 & $1: 3$ & 400 \\
\hline
\end{tabular}




\section{b) Secado}

Durante los experimentos de secado, la masa de las hojuelas húmedas fue medida utilizando una balanza analítica. Al inicio, el peso fue determinado cada minuto; y cuando el experimento avanzó y los cambios en la masa eran pequeños, cada cinco a diez minutos. Además, se evaluó la fracción de los dos solventes por el monitoreo de la composición del gas de flujo de salida utilizando un espectrofotómetro. Esta información hace posible observar como los solventes son secados individualmente.

Las hojuelas utilizadas en los experimentos de secado son las mismas hojuelas que fueron lixiviadas y por lo tanto las condiciones del material en el secado fueron las mismas que en la lixiviación (ver Tabla 1). El único parámetro adicional fue la temperatura de secado del gas. En todos los experimentos, la temperatura del gas fue $40{ }^{\circ} \mathrm{C}$, exceptuando una, donde la temperatura del gas fue $60^{\circ} \mathrm{C}$.

\section{Evaluación experimental}

a) Rendimiento del aceite y de la mezcla insecticida

Inmediatamente terminado el proceso de secado, se pesaron las hojuelas, se secaron totalmente en un horno y se volvieron a pesar. La última medición corresponde a la masa del sólido seco y así por medio de una diferencia de masa se obtuvo el contenido de humedad de las hojuelas.

Para determinar el rendimiento del aceite y de la mezcla insecticida, la mezcla de dos fases de la lixiviación, hexano conteniendo aceite y metanol conteniendo compuestos insecticidas, fue separada utilizando un embudo de separación. Luego, el hexano fue evaporado en un rotavapor y el residuo de aceite se pesó. El peso de la mezcla insecticida se calculó utilizando el siguiente balance de masa:

$$
m_{\text {insec ticida }}=m_{s, 0}-m_{s}-m_{\text {aceite }}
$$

donde $m_{s, 0}(\mathrm{~kg})$ es el peso inicial de las hojuelas, $m_{s}$ es el peso de las hojuelas secas $(\mathrm{kg})$ y $m_{\text {aceite }}$ es el peso del aceite $(\mathrm{kg})$.

b) Velocidad de secado

El procesamiento de los datos experimentales se hizo utilizando un programa escrito en MATLAB; el programa lee los datos experimentales obtenidos y construye la curva de velocidad de secado de acuerdo a la ecuación:

$$
N_{v}=-\frac{m_{s}}{A} \frac{d X}{d t}
$$

donde $N_{V}$ es la velocidad de secado por unidad de área $\left(\mathrm{kg} / \mathrm{m}^{2} \mathrm{~s}\right), X$ es la humedad de sólido base seca $(\mathrm{kg} / \mathrm{kg}), t$ es el tiempo (s) y $A$ es el área específica de secado $\left(\mathrm{m}^{2}\right)$.

c) Concentración del solvente de salida

Los resultados del espectrofotómetro tienen que ser transformado de volumen a concentración de acuerdo a:

$$
C_{i}=V_{i} \frac{\rho_{i}}{M_{i}} \frac{1}{V_{\text {celda }}}
$$


donde $C_{i}$ es la concentración del solvente $i\left(\mathrm{kmol} / \mathrm{m}^{3}\right), \rho_{i}$ es la densidad del solvente $i\left(\mathrm{~kg} / \mathrm{m}^{3}\right), M_{i}$ es el peso molecular del solvente $i(\mathrm{~kg} / \mathrm{kmol}), V_{i}$ es el volumen total del solvente $i\left(\mathrm{~m}^{3}\right)$, medido por el espectrofotómetro y $V_{\text {celda }}$ es el volumen de la celda que corresponde a $500 \mathrm{~mL}$. Los datos de densidades y pesos moleculares de los solventes fueron obtenidos en Green y Perry (2008).

\section{RESULTADOS Y DISCUSIÓN}

\section{Lixiviación}

a) Influencia del tiempo de lixiviación

La operación de lixiviación fue realizada durante 10, 20 y $30 \mathrm{~h}$. De acuerdo a los resultados (ver Tabla 2), a mayor tiempo de lixiviación mayor rendimiento de la mezcla de insecticida fue alcanzado. El rendimiento del aceite alcanzó aproximadamente el mismo valor independiente del tiempo de lixiviación. El aceite fue lixiviado más rápido que la mezcla insecticida. Esto indica que el equilibrio fue alcanzado más temprano con el aceite que con la mezcla insecticida. Los resultados descritos anteriormente concuerdan con los reportados por Martínez (2003).

Tabla 2 Influencia del tiempo de lixiviación.

\begin{tabular}{|c|c|c|c|c|c|c|c|}
\hline $\begin{array}{l}\text { Tiempo de } \\
\text { lixiviación } \\
\text { (h) }\end{array}$ & $\begin{array}{l}\text { Grosor } \\
\text { de la } \\
\text { hojuela } \\
(\mathrm{mm})\end{array}$ & $\begin{array}{c}\text { Relación de } \\
\text { volumen de los } \\
\text { solventes } \\
\text { (hexano:metanol) }\end{array}$ & $\begin{array}{l}\text { Volumen } \\
\text { total de } \\
\text { solventes } \\
(\mathrm{mL})\end{array}$ & $\begin{array}{c}\text { Aceite } \\
(\%)\end{array}$ & $\begin{array}{c}\text { Promedio } \\
(\%)\end{array}$ & $\begin{array}{c}\text { Insecticida } \\
(\%)\end{array}$ & $\begin{array}{c}\text { Promedio } \\
(\%)\end{array}$ \\
\hline 10 & 2.0 & $1: 2$ & 300 & 35.8 & 35.80 & 7.40 & 7.40 \\
\hline 20 & 2.0 & $1: 2$ & 300 & $\begin{array}{l}35.5 \\
35.3\end{array}$ & 35.40 & $\begin{array}{l}10.3 \\
11.0\end{array}$ & 10.65 \\
\hline 20 & 2.0 & $1: 3$ & 400 & $\begin{array}{l}33.9 \\
33.6\end{array}$ & 33.75 & $\begin{array}{l}13.1 \\
13.5\end{array}$ & 13.30 \\
\hline 30 & 2.0 & $1: 3$ & 400 & $\begin{array}{l}34.4 \\
34.0 \\
33.8 \\
34.4\end{array}$ & 34.15 & $\begin{array}{l}19.3 \\
18.8 \\
18.1 \\
18.0\end{array}$ & 18.55 \\
\hline
\end{tabular}

b) Influencia del grosor de las hojuelas

Cuando se utilizó hojuelas con un grosor de $0.7 \mathrm{~mm}$, el rendimiento del aceite fue notablemente mayor que cuando se utilizó un grosor de $2 \mathrm{~mm}$. El rendimiento de la mezcla de insecticida también fue mayor, pero no mucho como el del aceite (ver Tabla 3). Cuando las hojuelas son más delgadas, el área específica es más grande lo cual permite a los solventes lixiviar más aceite e insecticida. La trayectoria de la difusión también es más corta lo que resulta en una transferencia de masa más rápida.

Por otro lado, los resultados cuando se utiliza un grosor de hojuela de $0.7 \mathrm{~mm}$ son bastante incierto por causa de una cera blanca que aparece directamente después de la lixiviación; de que consiste la cera es desconocido. Probablemente sea puro aceite pero también podría ser una especie de mezcla de aceite y los dos solventes. Esto hace que los diferentes compuestos en la mezcla (hexano, metanol, aceite, insecticida y cera) sean difíciles de separar. 
Debido a la difícil separación, los resultados son pocos confiables, lo cual puede verse en la variación considerable de los rendimientos del aceite y de la mezcla insecticida en los experimentos donde el grosor $0.7 \mathrm{~mm}$ fue utilizado (ver Tabla 3).

Tabla 3 Influencia del grosor de las hojuelas.

\begin{tabular}{|c|c|c|c|c|c|c|c|}
\hline $\begin{array}{c}\text { Grosor } \\
\text { de la } \\
\text { hojuela } \\
(\mathrm{mm})\end{array}$ & $\begin{array}{l}\text { Tiempo de } \\
\text { lixiviación } \\
\text { (h) }\end{array}$ & $\begin{array}{c}\text { Relación de } \\
\text { volumen de los } \\
\text { solventes } \\
\text { (hexano:metanol) }\end{array}$ & $\begin{array}{l}\text { Volumen } \\
\text { total de } \\
\text { solventes } \\
(\mathrm{mL}) \\
\end{array}$ & $\begin{array}{c}\text { Aceite } \\
(\%)\end{array}$ & $\begin{array}{c}\text { Promedio } \\
(\%)\end{array}$ & $\begin{array}{l}\text { Insecticida } \\
(\%)\end{array}$ & $\begin{array}{c}\text { Promedio } \\
(\%)\end{array}$ \\
\hline 0.7 & 20 & $1: 2$ & 300 & $\begin{array}{l}42.9 \\
40.0 \\
47.0\end{array}$ & 43.30 & $\begin{array}{l}14.1 \\
18.4 \\
9.40\end{array}$ & 13.97 \\
\hline 2.0 & 20 & $1: 2$ & 300 & $\begin{array}{l}35.5 \\
35.3\end{array}$ & 35.40 & $\begin{array}{l}10.3 \\
11.0\end{array}$ & 10.65 \\
\hline 0.7 & 20 & $1: 3$ & 400 & $\begin{array}{l}40.9 \\
43.4\end{array}$ & 42.15 & $\begin{array}{l}15.5 \\
12.6\end{array}$ & 14.05 \\
\hline 2.0 & 20 & $1: 3$ & 400 & $\begin{array}{l}33.9 \\
33.6 \\
\end{array}$ & 37.50 & $\begin{array}{l}13.1 \\
13.5 \\
\end{array}$ & 13.30 \\
\hline
\end{tabular}

c) Influencia de la relación de volumen de los solventes y volumen total de solventes

En la Tabla 4 se puede observar que las diferentes relaciones de volumen de hexano y metanol tienen una influencia significativa en el rendimiento de la mezcla insecticida, mientras que en el caso del rendimiento del aceite solo varia ligeramente. El más alto rendimiento de la mezcla insecticida fue alcanzado cuando más metanol que hexano fue utilizado.

Tabla 4 Influencia de la relación de volumen de los solventes.

\begin{tabular}{cccccc}
\hline $\begin{array}{c}\text { Relación de } \\
\text { volumen de los } \\
\text { solventes } \\
\text { (hexano:metanol) }\end{array}$ & $\begin{array}{c}\text { Volumen } \\
\text { total de } \\
\text { solventes } \\
(\mathrm{mL})\end{array}$ & $\begin{array}{c}\text { Grosor de } \\
\text { hojuela } \\
(\mathrm{mm})\end{array}$ & $\begin{array}{c}\text { Tiempo de } \\
\text { lixiviación } \\
(\mathrm{h})\end{array}$ & $\begin{array}{c}\text { Aceite } \\
(\%)\end{array}$ & $\begin{array}{c}\text { Insecticida } \\
(\%)\end{array}$ \\
\hline $2: 1$ & 300 & 2 & 10 & 39.0 & 1.8 \\
$1: 1$ & 300 & 2 & 10 & 38.1 & 2.8 \\
$1: 2$ & 300 & 2 & 10 & 35.8 & 7.4 \\
\hline
\end{tabular}

El rendimiento del aceite fue aproximadamente el mismo independiente de la relación de volumen y volumen total de solventes. Una excepción se da cuando se utilizan volúmenes iguales de los dos solventes o más hexano que metanol, pero en esos casos los rendimientos de la mezcla insecticida fueron muy bajos para usarlos (ver Tabla 5). Por ejemplo, cuando se utiliza $100 \mathrm{~mL}$ de hexano el rendimiento de la mezcla de insecticida es más alto que con $150 \mathrm{~mL}$ de hexano y cuando se utiliza el más alto volumen total de solventes el rendimiento de la mezcla insecticida decrece (comparar los dos experimentos con la relación 1 a 2 cuando las hojuelas han sido lixiviadas por 20 h; ver Tabla 5).

Al parecer la adsorción ocurre preferentemente con el hexano más que con el metanol. Probablemente, cuando se usa el más alto volumen de solventes, el hexano ocupa el espacio donde se supone que el metanol debería lixiviar la mezcla de insecticida, el cual resulta en menos mezcla insecticida aunque más metanol esta en el sistema. 
Hasta ahora, se ha mostrado que la relación de volumen y el volumen total de solventes afectan el rendimiento del aceite y de la mezcla de insecticida. Analizando los resultados (ver Tabla 5), se observa que comparando el mismo volumen con diferentes relaciones da una grande diferencia en el rendimiento del aceite y de la mezcla insecticida, mientras que comparado la misma relación con diferentes volúmenes solamente da una pequeña diferencia del efecto total. De acuerdo a estos resultados, el parámetro que más afecta de los dos es la relación entre los solventes pero la influencia del volumen no es definitivamente insignificante.

Tabla 5 Influencia de la relación de volumen de los solventes y el volumen total de solventes.

\begin{tabular}{cccccccc}
\hline $\begin{array}{c}\text { Relación de } \\
\text { volumen de } \\
\text { solventes } \\
\text { (hexano:metanol) }\end{array}$ & $\begin{array}{c}\text { Volumen } \\
\text { total de } \\
\text { solventes } \\
(\mathrm{mL})\end{array}$ & $\begin{array}{c}\text { Tiempo de } \\
\text { lixiviación } \\
(\mathrm{h})\end{array}$ & $\begin{array}{c}\text { Grosor } \\
\text { de } \\
\text { hojuela } \\
(\mathrm{mm})\end{array}$ & $\begin{array}{c}\text { Aceite } \\
(\%)\end{array}$ & $\begin{array}{c}\text { Promedio } \\
(\%)\end{array}$ & $\begin{array}{c}\text { Insecticida } \\
(\%)\end{array}$ & $\begin{array}{c}\text { Promedio } \\
(\%)\end{array}$ \\
\hline $1: 1$ & 300 & 20 & 2 & 40.6 & 40.6 & 5.5 & 5.50 \\
$1: 2$ & 300 & 20 & 2 & 35.5 & 35.4 & 10.3 & 10.65 \\
$1: 2$ & 450 & 20 & 2 & 35.3 & 35.5 & 8.6 & 8.60 \\
$3: 4$ & 350 & 20 & 2 & 37.0 & 37.0 & 7.1 & 7.10 \\
$1: 3$ & 400 & 20 & 2 & 33.9 & 33.75 & 13.1 & 13.3 \\
$1: 2$ & 450 & 30 & 2 & 36.3 & 36.3 & 9.9 & 9.90 \\
& & & & 34.4 & & 19.3 & \\
$1: 3$ & 400 & 30 & 2 & 34.0 & 34.15 & 18.8 & 18.5 \\
& & & & 33.8 & & 18.0 & \\
\hline
\end{tabular}

\section{Comparación de los resultados de la lixiviación con estudios previos}

Las condiciones que producen el más alto rendimiento de la mezcla insecticida en este estudio (cuando la lixiviación simultánea fue utilizada) fueron cuando las hojuelas se lixiviaron por $30 \mathrm{~h}$, el grosor de hojuela fue $2 \mathrm{~mm}$ y la relación de hexano y metanol fue 1 a 3 (ver Tabla 5). Si las hojuelas de $0.7 \mathrm{~mm}$ hubiesen sido lixiviadas por $30 \mathrm{~h}$, probablemente hubiera resultado hasta en un mayor, o al menos el mismo, rendimiento de mezcla insecticida como cuando las hojuelas de $2 \mathrm{~mm}$ fueron lixiviadas por $30 \mathrm{~h}$.

Espinosa (2000) lixivió semillas molidas de nim en un extractor por lote multi-etapas a contracorriente. Las semillas fueron primeramente lixiviadas con hexano para extraer el aceite y después con etanol para extraer la mezcla insecticida (ver Tabla 6).

Martínez (2003) usó hojuelas de nim de diferentes grosores en un proceso de lixiviado de dos etapas en columnas. Primero, las hojuelas fueron lixiviadas por $20 \mathrm{~h}$ con hexano, para lixiviar el aceite y luego fueron secadas. Después de eso, las hojuelas fueron lixiviadas por $20 \mathrm{~h}$ con etanol, para lixiviar la mezcla insecticida y finalmente secadas nuevamente (ver Tabla 7).

Tabla 6 Resultados de Espinosa (2000).

\begin{tabular}{ccc}
\hline \multirow{2}{*}{$\begin{array}{c}\text { Semillas molidas } \\
\text { de nim }\end{array}$} & $\begin{array}{c}\text { Aceite } \\
(\%)\end{array}$ & $\begin{array}{c}\text { Insecticida } \\
(\%)\end{array}$ \\
\cline { 2 - 3 } & 32.4 & 11.4 \\
\hline
\end{tabular}


Tabla 7 Resultados de Martínez (2003).

\begin{tabular}{cccc}
\hline $\begin{array}{c}\text { Grosor de } \\
\text { hojuela } \\
(\mathrm{mm})\end{array}$ & $\begin{array}{c}\text { Tiempo de } \\
\text { Lixiviación } \\
(\mathrm{h})\end{array}$ & $\begin{array}{c}\text { Aceite } \\
(\%)\end{array}$ & $\begin{array}{c}\text { Insecticida } \\
(\%)\end{array}$ \\
\hline 2.0 & $2 \times 20$ & 41.40 & 10.37 \\
0.7 & $2 \times 20$ & 43.26 & 16.73 \\
\hline
\end{tabular}

Comparando los rendimientos del aceite y de la mezcla insecticida del presente estudio con el proceso por lote realizado por Espinosa (2000), se observa que ambos rendimientos fueron mayores cuando se usó la lixiviación simultánea. Cuando comparamos los resultados con el proceso de dos etapas de Martínez (2003), se notó que para las hojuelas de $2 \mathrm{~mm}$ el rendimiento de la mezcla insecticida fue mucho mayor en la lixiviación simultánea y el rendimiento del aceite fue menor. Para las hojuelas de $0.7 \mathrm{~mm}$, el rendimiento de aceite fue comparable pero el rendimiento de la mezcla insecticida fue menor.

Debido a que el hexano y metanol son miscibles, no fue posible usar estos dos solventes en la lixiviación simultánea, ya que después la separación hubiera sido muy complicada. Por esta razón, el metanol fue utilizado en lugar de etanol por tener propiedades similares. El hexano y metanol son completamente inmiscibles. Cuál de los solventes, etanol o metanol, tiene la más alta capacidad de lixiviar la mezcla insecticida es desconocida. Para conocer lo último es necesario evaluar las propiedades del solvente en futuros estudios.

Los diferentes procedimientos de lixiviación y combinaciones de solventes hacen que los resultados sean difíciles de comparar. No es posible establecer si es la manera de lixiviación o el cambio de solvente que afecta mayormente los rendimientos.

Cuando se usó una mezcla de hexano y metanol en la lixiviación simultánea resultó en un mayor rendimiento de la mezcla insecticida para las hojuelas de $2 \mathrm{~mm}$, que usando hexano y etanol uno por uno en el proceso de dos etapas. Para las hojuelas de $0.7 \mathrm{~mm}$, el rendimiento de la mezcla insecticida es mayor en el proceso de dos etapas. El rendimiento del aceite es mayor en el proceso de dos etapas cuando se usó hojuelas de $2 \mathrm{~mm}$ y aproximadamente el mismo usando el grosor de $0.7 \mathrm{~mm}$.

Hasta ahora, el tiempo total del proceso no ha sido considerado. La lixiviación simultánea, comparada con el proceso de dos etapas, reduce una lixiviación y una etapa de secado y en su lugar se añade una etapa de separación. Utilizando las hojuelas de $2 \mathrm{~mm}$, en la lixiviación simultánea, el rendimiento de la mezcla insecticida es más alto que en el proceso de dos etapas cuando las hojuelas fueron lixiviadas por $20 \mathrm{~h}$ y casi el doble cuando las hojuelas fueron lixiviadas por $30 \mathrm{~h}$. Utilizando la lixiviación simultánea el tiempo total del proceso es reducido y el rendimiento de la mezcla insecticida es mayor. Utilizando hojuelas de $0.7 \mathrm{~mm}$, el rendimiento de la mezcla insecticida es mayor todavía en el proceso de dos etapas que en la lixiviación simultánea pero las hojuelas solo fueron lixiviadas por $20 \mathrm{~h}$. Si las hojuelas fueran lixiviadas por $30 \mathrm{~h}$, el rendimiento de la mezcla insecticida sería ciertamente más superior que en el proceso de dos etapas y el tiempo total del proceso sería reducido.

\section{Secado}

a) Influencia de la temperatura de secado

Como es de esperarse, el tiempo de secado se reduce al aumentar la temperatura del gas. En la Fig. 3, se observan las curvas de secado a $40{ }^{\circ} \mathrm{C}$ y $60{ }^{\circ} \mathrm{C}$. En la Fig. 4, se muestran las curvas de velocidad de secado. En las curvas de velocidad de secado, no existe el periodo de velocidad constante y solo un 
periodo de velocidad decreciente se observa. Un periodo de velocidad constante indicaría que la transferencia externa de calor y masa están limitando el proceso de secado. Las curvas de velocidad de secado, en la Fig. 4, muestran que una alta velocidad de secado ocurre a cierto contenido de humedad empleando una temperatura alta, que cuando se emplea una temperatura más baja. Utilizando una temperatura mayor hace que el gas se sature menos, lo que hace posible secar más rápido las hojuelas. Las curvas de secado y de velocidad de secado de las restantes muestras de hojuelas lixiviadas muestran las mismas tendencias que las Figs. 3 y 4. Sin embargo, estos resultados no fueron posibles compararlos ya que el aire de secado no tuvo una temperatura uniforme ni el contenido de humedad del lecho de hojuelas.

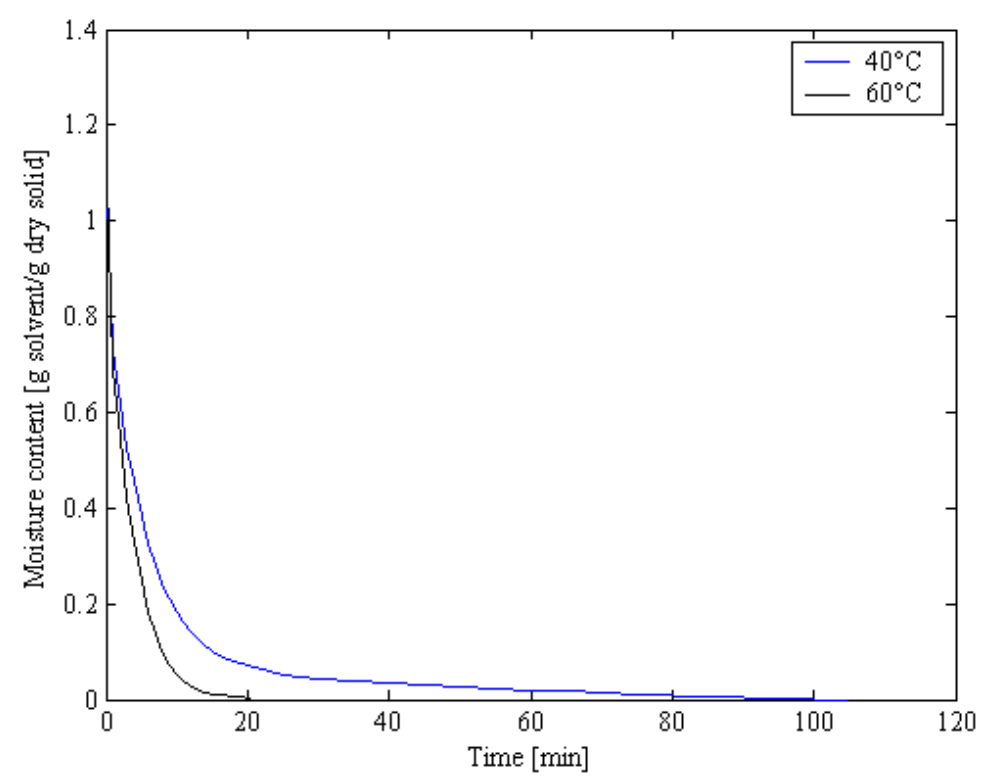

Fig. 3 Curvas de secado a $40{ }^{\circ} \mathrm{C}$ y $60^{\circ} \mathrm{C}$.



Fig. 4 Curvas de velocidad de secado a $40{ }^{\circ} \mathrm{C}$ y $60{ }^{\circ} \mathrm{C}$. 
b) Contenido de humedad

Al inicio del proceso de secado, el contenido de humedad es alto cuando se emplean los grosores de hojuelas más finos. La hojuelas con los grosores más grandes tienen un contenido de humedad de aproximadamente $1.1 \mathrm{a} 1.3 \mathrm{~g}$ solvente/g hojuelas secas cuando las hojuelas fueron lixiviadas por $20 \mathrm{~h}$; y 1.2 a $1.7 \mathrm{~g}$ solvente/g hojuelas secas cuando las hojuelas fueron lixiviadas por $30 \mathrm{~h}$. El contenido de humedad, cuando se emplean los grosores de hojuelas más finos, fue de 1.8 a $2.0 \mathrm{~g}$ solvente/g hojuelas secas cuando las hojuelas fueron lixiviadas por $20 \mathrm{~h}$.

Probablemente, la interacción entre las hojuelas y el líquido es más grande con un área específica mayor. Más compuestos de aceite e insecticida fueron lixiviados cuando se empleó un grosor de hojuelas de 0.7 mm que cuando se empleó un grosor de hojuelas de $2 \mathrm{~mm}$, de acuerdo a los resultados mostrados en la Tabla 8. Además, parece posible realizar una conexión entre el contenido de humedad al inicio del proceso de secado y los rendimientos de los compuestos de aceite e insecticida extraídos. Entre más compuestos de aceite e insecticida fueron extraídos (lixiviados), mayor es el contenido de humedad de las hojuelas al inicio del proceso de secado.

Tabla 8 Resumen de los resultados de la lixiviación.

\begin{tabular}{|c|c|c|c|c|c|c|c|}
\hline $\begin{array}{l}\text { Tiempo de } \\
\text { lixiviación } \\
\text { (h) }\end{array}$ & $\begin{array}{l}\text { Grosor } \\
\text { de la } \\
\text { hojuela } \\
\text { (mm) }\end{array}$ & $\begin{array}{c}\text { Relación de } \\
\text { volumen de los } \\
\text { solventes } \\
\text { (hexano:metanol) }\end{array}$ & $\begin{array}{l}\text { Volumen } \\
\text { total de } \\
\text { solventes } \\
(\mathrm{mL})\end{array}$ & $\begin{array}{c}\text { Aceite } \\
(\%)\end{array}$ & $\begin{array}{c}\text { Promedio } \\
(\%)\end{array}$ & $\begin{array}{c}\text { Insecticida } \\
(\%)\end{array}$ & $\begin{array}{c}\text { Promedio } \\
(\%)\end{array}$ \\
\hline 10 & 2 & $1: 1$ & 300 & 38.1 & 38.1 & 2.80 & 2.80 \\
\hline 10 & 2 & $1: 2$ & 300 & 35.8 & 35.8 & 7.40 & 7.40 \\
\hline 10 & 2 & $2: 1$ & 300 & 39.0 & 39.0 & 1.80 & 1.80 \\
\hline 20 & 2 & $1: 1$ & 300 & 40.6 & 40.6 & 5.50 & 5.50 \\
\hline 20 & 2 & $1: 2$ & 300 & $\begin{array}{l}35.5 \\
35.3\end{array}$ & 35.4 & $\begin{array}{l}10.3 \\
11.0\end{array}$ & 10.65 \\
\hline 20 & 2 & $1: 2$ & 450 & 35.5 & 35.5 & 8.60 & 8.60 \\
\hline 20 & 2 & $3: 4$ & 350 & 37.0 & 37.0 & 7.10 & 7.10 \\
\hline 20 & 2 & $1: 3$ & 400 & $\begin{array}{l}33.9 \\
33.6\end{array}$ & 33.75 & $\begin{array}{l}13.1 \\
13.5\end{array}$ & 13.3 \\
\hline 30 & 2 & $1: 2$ & 450 & $\begin{array}{l}36.3 \\
34.4\end{array}$ & 36.3 & $\begin{array}{l}9.90 \\
19.3\end{array}$ & 9.90 \\
\hline 30 & 2 & $1: 3$ & 400 & $\begin{array}{l}34.0 \\
33.8 \\
34.4 \\
42.9\end{array}$ & 34.15 & $\begin{array}{l}18.8 \\
18.1 \\
18.0 \\
14.1\end{array}$ & 18.55 \\
\hline 20 & 0.7 & $1: 2$ & 300 & $\begin{array}{l}40.0 \\
47.0\end{array}$ & 43.3 & $\begin{array}{l}18.4 \\
9.40\end{array}$ & 13.97 \\
\hline 20 & 0.7 & $1: 3$ & 400 & $\begin{array}{l}40.9 \\
43.4\end{array}$ & 42.15 & $\begin{array}{l}15.5 \\
12.6\end{array}$ & 14.05 \\
\hline
\end{tabular}

Después del proceso de secado en la columna, las hojuelas se introdujeron en un horno con el objetivo de secar el contenido de humedad restante. El contenido de humedad en las hojuelas, después del secado en la columna, vario tanto en los experimentos con condiciones diferentes así como en experimentos bajo las mismas condiciones (ver Tabla 9). Las diferencias pueden ser debidas a que el aire de secado entrante no tenía la misma humedad inicial en todos los experimentos. El contenido de humedad no removido en las hojuelas, después de secado, depende de la humedad de inicial del gas de secado entrante a la columna. El secado de las hojuelas ocurre hasta que el equilibrio entre el aire de secado y las hojuelas aparece. Más 
allá de esta etapa, no existe ninguna fuerza impulsora para secar aún más las hojuelas. Un flujo de gas inicial más humidificado resulta en que el equilibrio se produce con una mayor concentración de solventes que quedan en las hojuelas. Con un flujo de gas inicial menos humidificado el equilibrio ocurre a un contenido de humedad inferior y en una etapa posterior, lo cual permite que las hojuelas estén más secas al final del proceso de secado.

Tabla 9 Contenidos de humedad al inicio y al final del proceso de secado.

\begin{tabular}{|c|c|c|c|c|c|c|}
\hline $\begin{array}{l}\text { Grosor } \\
\text { de la } \\
\text { hojuela } \\
(\mathrm{mm})\end{array}$ & $\begin{array}{l}\text { Tiempo de } \\
\text { lixiviación } \\
\text { (h) }\end{array}$ & $\begin{array}{c}\text { Relación de } \\
\text { volumen de los } \\
\text { solventes } \\
\text { (hexano:metanol) }\end{array}$ & $\begin{array}{l}\text { Volumen } \\
\text { total de } \\
\text { solventes } \\
\text { (mL) }\end{array}$ & $\begin{array}{l}\text { Contenido } \\
\quad \text { de } \\
\text { humedad } \\
\text { inicial }(\mathrm{g} / \mathrm{g})\end{array}$ & $\begin{array}{l}\text { Contenido } \\
\text { de } \\
\text { humedad } \\
\text { final }(\mathrm{g} / \mathrm{g})\end{array}$ & $\begin{array}{l}\text { Contenido de } \\
\text { humedad } \\
\text { removido } \\
(\mathrm{g} / \mathrm{g})\end{array}$ \\
\hline \multirow[b]{2}{*}{2} & \multirow{2}{*}{10} & \multirow{2}{*}{$1: 1$} & \multirow{2}{*}{300} & 1.06 & - & $1.06^{1}$ \\
\hline & & & & 1.09 & - & $1.09^{1}$ \\
\hline \multirow{2}{*}{2} & \multirow{2}{*}{10} & \multirow{2}{*}{$1: 2$} & \multirow{2}{*}{300} & 0.93 & - & $0.93^{1}$ \\
\hline & & & & 0.99 & - & $0.99^{1}$ \\
\hline 2 & 20 & $1: 1$ & 300 & 1.30 & 0.07 & 1.23 \\
\hline \multirow{2}{*}{2} & \multirow{2}{*}{20} & \multirow{2}{*}{$1: 2$} & \multirow{2}{*}{300} & 1.28 & 0.05 & 1.24 \\
\hline & & & & 1.19 & 0.09 & 1.11 \\
\hline 2 & 20 & $1: 2$ & 450 & 1.13 & 0.05 & 1.08 \\
\hline \multirow{2}{*}{2} & \multirow{2}{*}{20} & \multirow{2}{*}{$1: 3$} & \multirow{2}{*}{400} & 1.29 & 0.09 & 1.19 \\
\hline & & & & 1.35 & 0.09 & 1.25 \\
\hline \multirow[t]{2}{*}{2} & \multirow{2}{*}{30} & \multirow[t]{2}{*}{$1: 2$} & \multirow{2}{*}{450} & 1.28 & 0.07 & 1.17 \\
\hline & & & & 1.66 & 0.21 & 1.46 \\
\hline \multirow{3}{*}{2} & \multirow{3}{*}{30} & \multirow{3}{*}{$1: 3$} & \multirow{3}{*}{400} & 1.62 & 0.18 & 1.44 \\
\hline & & & & 1.53 & 0.18 & 1.35 \\
\hline & & & & 1.43 & 0.10 & 1.33 \\
\hline \multirow{2}{*}{0.7} & \multirow{2}{*}{20} & \multirow{2}{*}{$1: 2$} & \multirow{2}{*}{300} & 2.01 & 0.11 & 1.90 \\
\hline & & & & 1.90 & 0.09 & 1.81 \\
\hline \multirow{2}{*}{0.7} & \multirow{2}{*}{20} & \multirow{2}{*}{$1: 3$} & \multirow{2}{*}{400} & 1.93 & 0.09 & 1.85 \\
\hline & & & & 1.85 & 0.04 & 1.81 \\
\hline
\end{tabular}

${ }^{1}$ Las hojuelas no fueron introducidas en el horno después del secado, debido a estos los valores son demasiados altos.

El contenido de humedad restante también depende de cómo el lecho está empaquetado, por ejemplo, cuántos de los poros están bloqueados por otras hojuelas. Cuando los poros están bloqueados, el flujo de aire no puede llegar a los solventes. El lecho nunca esta empaquetado de la misma manera exacta, lo que significa que las diferentes cantidades de solventes son inalcanzables para el flujo de aire. En el horno, donde el área de transferencia de masa cambia, las hojuelas se secan en su totalidad.

c) Influencia del tiempo de lixiviación sobre el secado

Entre más tiempo las hojuelas se lixivian, las hojuelas se vuelven más porosas. Una hojuela más porosa significa una trayectoria de difusión más larga, pero también que el líquido se puede transportar más fácilmente. Estos dos fenómenos se contrarrestan entre sí. Comparando los experimentos de lixiviación bajo las mismas condiciones, excepto por el tiempo de lixiviación, se determinara cuál de los dos fenómenos tiene mayor efecto.

A partir de los datos experimentales, se determinó que la comparación entre los diferentes tiempos de lixiviación es imposible, ya que el tiempo de secado en experimentos similares varía demasiado. Las variaciones en el tiempo de secado se discuten en la siguiente sección. 


\section{Nyberg et al.}

\section{d) Variación en el tiempo de secado}

Al inicio del proceso de secado, la humedad tiene un camino de difusión corto para llegar a la interface, y el contenido de humedad decrece muy rápidamente con el tiempo. A medida que el secado procede, el camino de difusión se hace más largo y el secado del contenido de humedad restante es lento. Estos resultados experimentales concuerdan con la teoría. Sin embargo, para los experimentos repetidos se observó una variación considerable en el tiempo de secado. Por ejemplo, en el experimento repetido cuatro veces, con las hojuelas de un grosor de $2 \mathrm{~mm}$ y lixiviadas durante 30 horas con una relación de hexano y metanol de tres a uno, se requirió completamente diferentes tiempos de secado para alcanzar el mismo contenido de humedad (ver Fig. 5). Para secar las hojuelas hasta $0.4 \mathrm{~g}$ solvente/g hojuelas secas, todos los experimentos reportan tiempos de secado similares. Sin embargo, después de los $0.4 \mathrm{~g}$ solvente/g hojuelas secas las cuervas de secado toman diferentes formas. En dos de los experimentos, el contenido de humedad continúa disminuyendo rápidamente, mientras que en los otros dos los cambios en el contenido de humedad con respecto al tiempo son muy pequeños. Estas variaciones dificultan las conclusiones sobre el tiempo de secado.

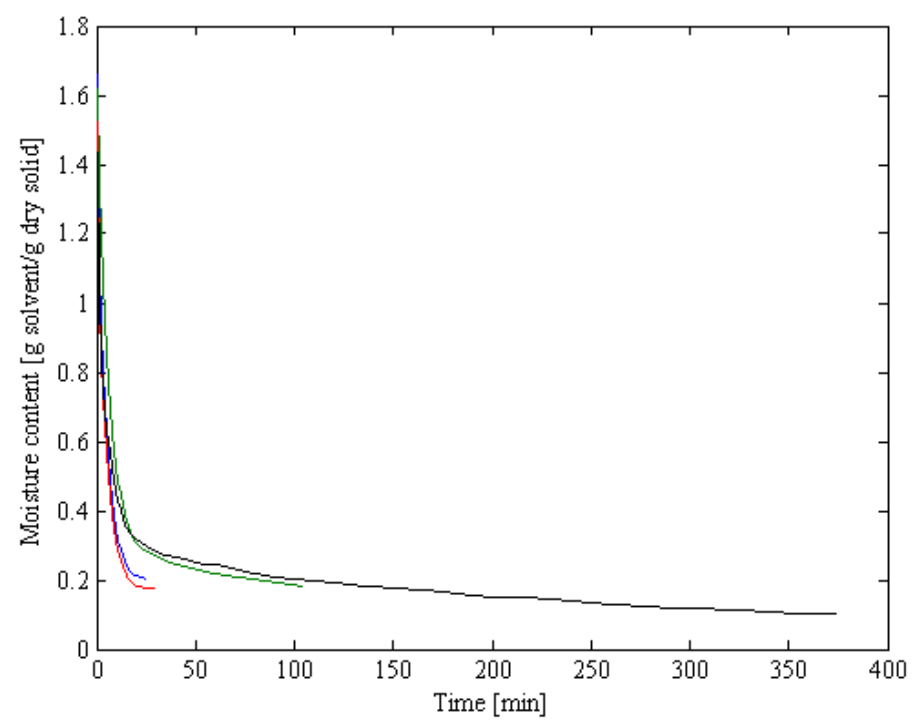

Fig. 5 Variaciones en el tiempo de secado.

El contenido de humedad de las hojuelas al inicio del proceso de secado afecta el tiempo de secado, pero esto no es seguramente la razón de la gran variación en los tiempos de secado. La razón podría ser debido a muchos factores, pero la razón principal es, probablemente, debido al aire entrante a la columna que tuvo diferente humedad. La columna de absorción no se regeneró antes de cada experimento. Esto da como resultado equilibrio diferente entre el aire entrante y las hojuelas húmedas. Un flujo de aire entrante humidificado resulta en un mayor contenido de humedad de equilibrio en las hojuelas. Más allá de esta etapa, el secado se detuvo. El proceso es muy difícil de analizar ya que la humedad en el aire es un solvente adicional. Otra razón importante para las diferencias podría ser la balanza analítica del equipo experimental.

e) Proceso de secado en la columna

De acuerdo a las curvas de perfiles de temperatura (no incluidas en el artículo), los experimentos de secado fueron principalmente realizados isotérmicamente, excepto al inicio del proceso de secado donde las temperaturas se reducen algunos grados. 


\section{Nyberg et al.}

Teoréticamente, el secado debe ser isotérmico hasta que las hojuelas estén totalmente secas, excepto al inicio del proceso. Debido a que la entrada del aire está en la parte inferior de la columna, las hojuelas cercanas al fondo son las primeras en secarse y el aire se satura antes de la salida de la columna. Un frente que separa la parte superior saturada y húmeda con la parte inferior seca aparece a través de la columna a medida que avanza el secado. Cuando el frente alcanza la parte superior de la columna y todas las hojuelas están secas, la temperatura del flujo de aire de salida aumenta debido que la fase liquida no está presente, por lo tanto no hay demanda de energía para la evaporación de humedad.

Cuando las hojuelas son colocadas en la columna y el secado inicia, la temperatura a la entrada de la columna disminuye. Este descenso de la temperatura se produce cuando el flujo de aire caliente y no saturado se encuentra con la fase liquida fría dentro de las hojuelas. La energía en el flujo de aire no es suficiente para evaporar el líquido, y por lo tanto la energía es tomada de las hojuelas. La temperatura de las hojuelas disminuye y el calor es transferido desde las paredes de la columna hacia las hojuelas, mientras la temperatura de las paredes de la columna disminuye. El flujo de aire entrante se enfría, lo que resulta en que la temperatura del flujo del aire entrante se reduce.

f) Medición de la concentración del gas de salida durante el secado

Para la determinación de las concentraciones del gas de salida se utilizó un espectrofotómetro, el cual proporcionó información de la composición del gas a diferentes tiempos. Con la información recolectada es posible determinar el contenido de humedad restante y la velocidad de secado para cada uno de los componentes de la mezcla, a cualquier intervalo de tiempo durante el proceso de secado. Idealmente, la curva de concentración debería comenzar en cero al momento que el secado inicia, al instante aumentar hasta un máximo, y luego a medida que avanza el secado disminuye gradualmente hasta cero otra vez. Datos de los tres primeros experimentos de secado se muestran en las Figs. 6-8. Las primeras mediciones en cada experimento no fue posible medirlas. Las variaciones en la concentración de los solventes que pasan a través del escáner fueron muy altas, lo que resulta en picos al inicio del secado donde la concentración es mucho mayor que al final. A cualquier tiempo, se detectó más metanol que hexano, independientemente de la relación de solvente empleada.

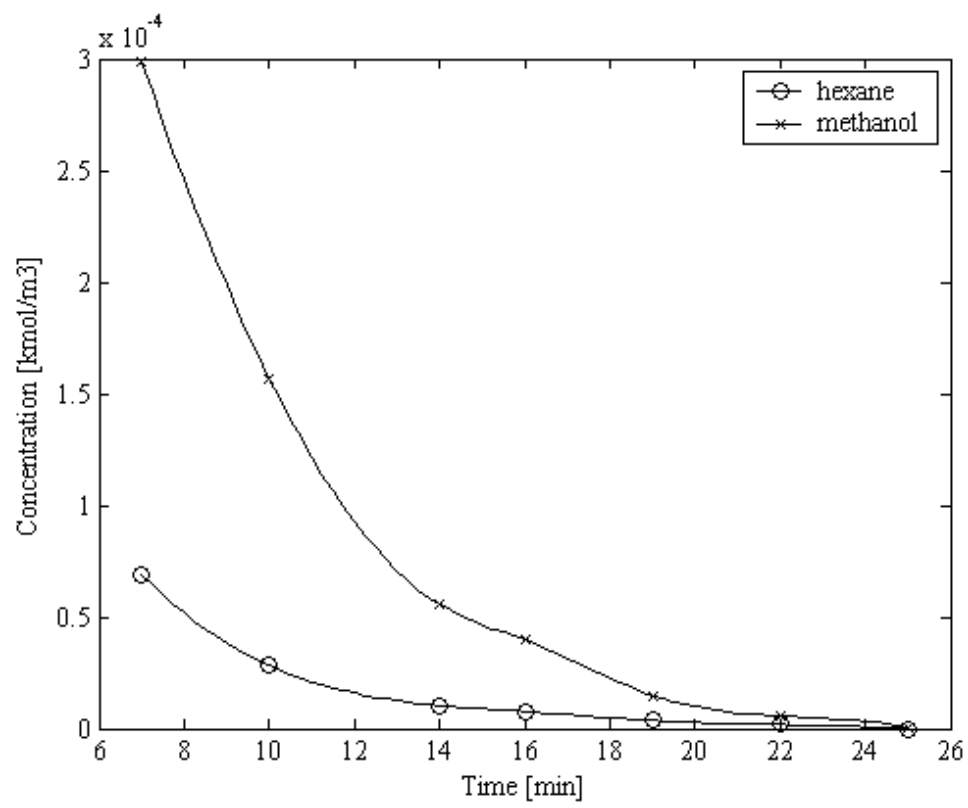

Fig. 6 Mediciones de la concentración del gas de salida durante el secado. 10 h de lixiviación, 2 mm de grosor de hojuela y 1:1 de relación de solvente hexano:metanol. 


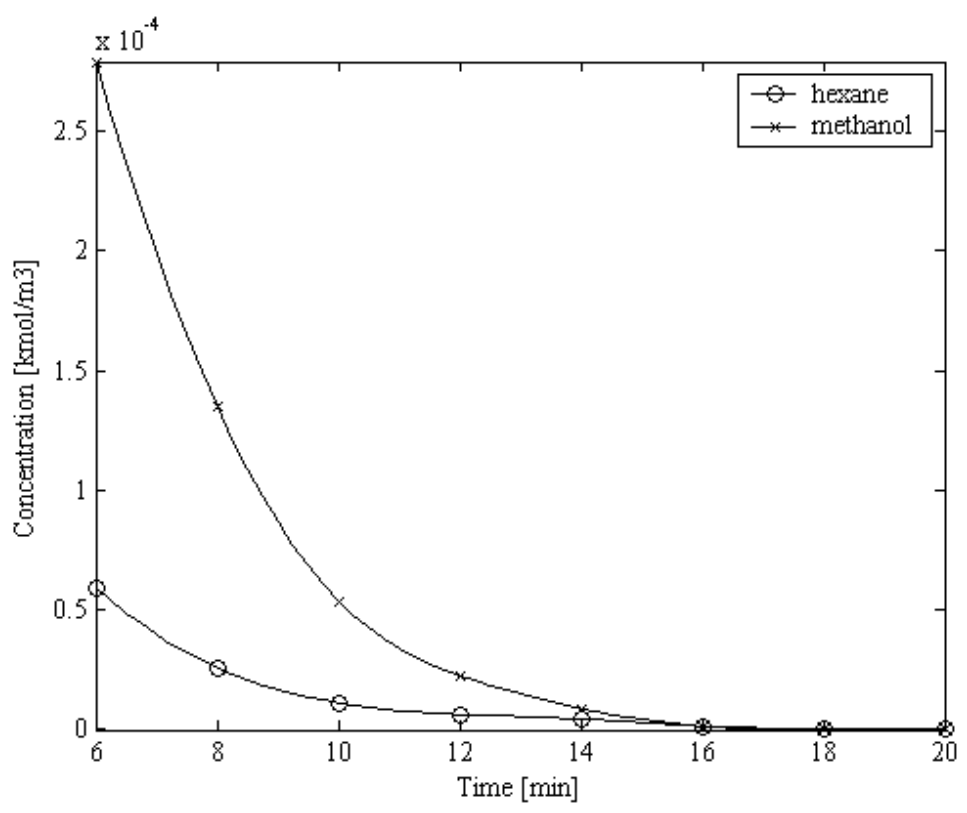

Fig. 7 Mediciones de la concentración del gas de salida durante el secado. $10 \mathrm{~h}$ de lixiviación, $2 \mathrm{~mm}$ de grosor de hojuela y 1:2 de relación de solvente hexano:metanol.

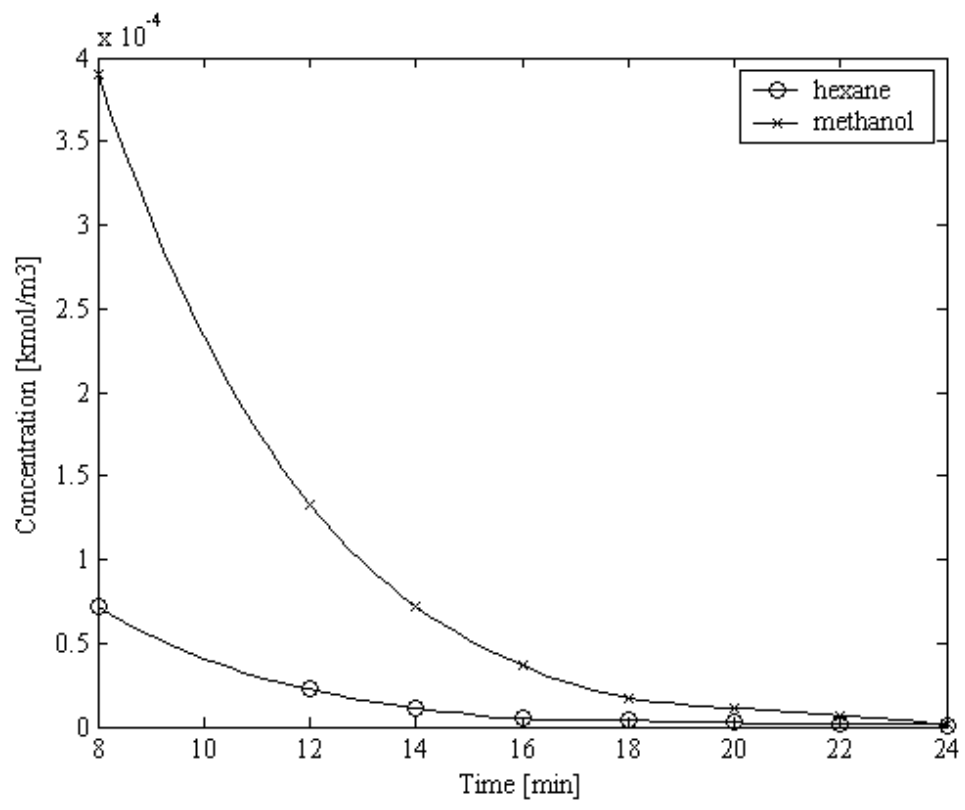

Fig. 8 Mediciones de la concentración del gas de salida durante el secado. 10 h de lixiviación, 2 mm de grosor de hojuela y 2:1 de relación de solvente hexano:metanol.

\section{CONCLUSIONES}

Los parámetros estudiados en la parte de lixiviación demostraron tener una gran influencia sobre el rendimiento de compuestos insecticidas, pero la influencia de la relación de solventes fue la más importante. Se demostró que más metanol que hexano tuvo que ser utilizado para obtener un alto rendimiento de compuestos insecticidas. El rendimiento de aceite fue influenciado principalmente por el 


\section{Nyberg et al.}

grosor de las hojuelas. Se alcanzó el rendimiento más alto de insecticida cuando hojuelas de $2 \mathrm{~mm}$ fueron lixiviadas durante $30 \mathrm{~h}$, empleando una relación de volumen de hexano y metanol de 1 a 3 , y un volumen total de solventes de $400 \mathrm{~mL}$. Este experimento se llevó a cabo cuatro veces y el rendimiento promedio de compuestos insecticidas fue de $18.55 \%$. Este es un porcentaje de rendimiento mayor en comparación tanto con el proceso por lotes así como el proceso de dos etapas.

La lixiviación con la mezcla de solventes polar/no-polar, en comparación con el proceso por lotes y el proceso de dos etapas, resulta en un mayor rendimiento de compuestos insecticidas en un tiempo de proceso más corto, empleando un grosor de hojuelas de $2 \mathrm{~mm}$. Para el grosor de hojuelas más pequeño, $0.7 \mathrm{~mm}$, se alcanzó un rendimiento de compuestos insecticidas del $2 \%$ menor en comparación al proceso de dos etapas en la mitad del tiempo total del proceso, pero los resultados tuvieron una amplia variación debido a que apareció una cera blanca que hizo la separación después de la lixiviación muy difícil. Por esta razón, las hojuelas de $0.7 \mathrm{~mm}$ no fueron lixiviadas durante más de $20 \mathrm{~h}$.

En la parte de secado, lo que puede afirmarse es que la temperatura del flujo de aire entrante tiene influencia en el proceso. Una temperatura mayor resulta en un tiempo de secado corto y en una velocidad de secado alta a cierto contenido de humedad. Conclusiones sobre el tiempo de secado o la velocidad de secado no son posibles de realizar a partir de los resultados de los experimentos. Las variaciones en el tiempo de secado son demasiadas grandes y la velocidad de secado parece ser independiente de las condiciones de la etapa de lixiviación anterior.

\section{AGRADECIMIENTOS}

Los autores agradecen a la Agencia Sueca de Cooperación Internacional para el Desarrollo (Asdi) por el apoyo financiero brindado para la realización de este trabajo.

\section{REFERENCIAS}

Angulo-Escalante, M.A., A.A. Gardea-Béjar, R. Vélez de la Rocha, R.S. García-Estrada, A. CarrilloFasio, C. Cháidez-Quiroz y J.I. Partida-López (2004). Contenido de Azadiractina A en Semillas de Nim (Azadirachta indica A. Juss) Colectadas en Sinaloa, México. Revista Fitotecnia Mexicana, Vol. 27, No. 4, pp. 305-311.

Biswas, K., I. Chattopadhyay, R.K. Banerjee and U. Bandyopadhyay (2002). Biological Activities and Medicinal Properties of Neem (Azadirachta indica). Current Science, Vol. 82, No. 11, pp. 1336-1345.

Espinosa, R. (2000). Leaching and Drying Process Steps in the Manufacture of a Botanical Insecticide from Ground Neem Seeds. Licentiate Treatise. Department of Chemical Engineering and Technology, Royal Institute of Technology (KTH). Stockholm, Sweden.

Green, D.W. y R.H. Perry (2008). Perry's Chemical Engineers' Handbook. 8ta Ed., pp. 2.28-2.46. McGraw-Hill Professional. Nueva York, Estados Unidos.

Koul, O., M.B. Isman y C.M. Ketkar (1990). Properties and Uses of Neem, Azadirachta indica. Canadian Journal of Botany, Vol. 68, No. 1, pp. 1-11.

Martínez, C. (2003). Estudio Experimental y Modelación del Proceso de Lixiviación de Hojuelas de Nim en Columnas. Informe Técnico. Departamento de Ingeniería Química y Biotecnología, Universidad de Chile. Santiago, Chile. 
Martínez, C., J.M. Casas y J. Martínez (1998). Extraction Kinetics of Neem Seed Flakes. In Proceedings of the 18th Inter-American Congress of Chemical Engineering (IACChE'98). San Juan, Puerto Rico.

Martínez-Pavón, C. (2006). Producción de Insecticida a partir de la Semilla de Nim: Avances en la Investigación de la Operación de Lixiviación. Nexo, Vol. 19, No. 1, pp. 43-49.

Persson, J. y J. Sjöberg (1998). Leaching of Neem Seed Flakes. Master's Thesis. Department of Chemical Engineering and Technology, Royal Institute of Technology (KTH). Stockholm, Sweden. 